WellBeing International

WBI Studies Repository

2010

\title{
Vigilance and Antipredator Responses of Caribbean Reef Squid
}

Jennifer A. Mather

University of Lethbridge

Follow this and additional works at: https://www.wellbeingintlstudiesrepository.org/acwp_asie

Part of the Animal Studies Commons, Behavior and Ethology Commons, and the Comparative Psychology Commons

\section{Recommended Citation}

Mather, J. A. (2010). Vigilance and antipredator responses of Caribbean reef squid. Marine and Freshwater Behaviour and Physiology, 43(5), 357-370.

This material is brought to you for free and open access by WellBeing International. It has been accepted for inclusion by an authorized administrator of the WBI Studies Repository. For more information, please contact wbisr-info@wellbeingintl.org.

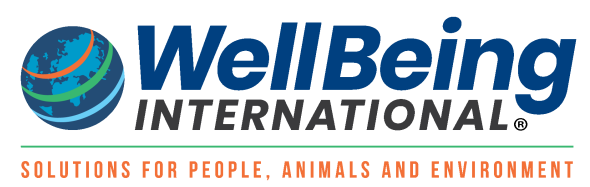




\title{
Vigilance and antipredator responses of Caribbean reef squid
}

Jennifer A. Mather

The University of Lethbridge

\section{KEYWORDS}

squid; Sepioteuthis sepioidea; behaviour; vigilance; antipredator responses

\begin{abstract}
Antipredator responses, especially those of open-ocean squid, have been seldom studied in the natural environment. Sepioteuthis sepioidea, observed by snorkellers near the shore in early morning/late afternoon, produced an average of eight moves of over $1 \mathrm{~m}$ per hour, apparently mostly antipredator behaviours. Close approaches by herbivorous parrotfish elicited no response in $74 \%$ of encounters; otherwise, squid produced agonistic zebra stripes or startle-mantle-dots skin patterns. Predatory bar jack fish caused flight but not zebra displays, and squid usually paled and fled quickly (66\%) from snapper. The speed of approach was the best predictor for flight and display responses to snapper, but for bar jack and parrotfish, the relative fish size and distance were the predictors for escalated responses. Paired dorsolateral mantle dots were produced when squid approached the sea bottom or hunted outside the group and in reaction to fish approaches; $56 \%$ of these were to the very common parrotfish. Reactive pairs of spots were selected from four possible mantle locations and they were significantly likely to be directional towards fish, presumably as startle/warning, but not directed towards conspecifics as indicators of predator presence. Thus the evasion techniques of the cephalopods and their ability to produce different display patterns on the skin show how an intelligent animal modifies an otherwise simple decision about which pairs of spots to select from four possible mantle locations and whether to flee from potentially dangerous animals.
\end{abstract}

\section{Introduction}

Antipredator behaviour is a complex set of responses along the predatory sequence, including camouflage or concealment, flight, and even predator inspection (Dugatkin 2004; Caro 2005). In addition, animals must balance predator avoidance with other influences such as the need to feed, availability of shelter and barriers to perception of an approaching threat. Because of this set of pressures, studies in conditions with the fewest of these situational variables mentioned above are useful if one is to evaluate what Ydenberg and Dill (1986) describe as decision making in the context of the economics of flight. Squid that swim in the open water with no proximity to land are at risk from predators in all directions but have no barriers to vision and no place to take shelter. This should eliminate concealment and camouflage as choices and make the response choice, to stay or to leave, simpler. 
Responses are complicated if animals, for instance squirrels (Lima and Valone 1986) and blue tits (Lendrem 1983), must balance vigilance with the immediate necessity to forage. Seeking shelter may also influence vigilance decisions as it does for crayfish (Stein and Magnusson 1976). Cover is important in the different vigilance behaviours of degu rodents (Ebensperger and Hurtado 2005) and the extent that camouflage matches the background influences when lizards will flee (Heatwole 1968). Studies in physically simple open areas such as grassland, in which deer, for instance, are faced with coyotes (Lingle and Wilson 2001) and emus are approached by humans (Boland 2003), are advantageous. Potential prey such as fathead minnows may also track predator risk over time (Ferrari and Chivers 2006). The simplicity of the physical environment for squid in midwater may also simplify the tradeoff of energy expenditure and safety that Ydenberg and Dill (1986) discuss. These authors suggest that flight distance will vary with changes in approach velocity, distance to cover and escape velocity - the last two of which are irrelevant to the present situation - as well as predator species.

Cephalopods, which are mollusks with no protective skeleton or shell in which to take refuge, are particularly vulnerable to predators. One device by which they reduce this risk is their sophisticated skin display system (Messenger 2001), which possesses chromatophores of red, yellow and brown as well as deeper reflective leucophores and iridophores which return green wavelengths or the ambient pattern of colours in the background. Such a system can be proactive when it blocks detection by predators, as the excellent camouflage in benthic octopuses (Packard 1995) and cuttlefish (Hanlon and Messenger 1988) simply prevents potential predators from noticing them. This camouflage is used to a lesser extent in squid (though see Moynihan and Rodaniche 1982). Complex camouflage is less useful for squid in the open sea, although they have countershading (Messenger 2001) but the ability of the display system to change patterns in less than $30 \mathrm{~ms}$ and across less than a square millimetre of skin allows the squid to produce sudden total changes in appearance like those of the octopus (Hanlon et al. 1999). Only young of Sepioteuthis sepioidea squid commonly use these major changes in appearance (Moynihan and Rodaniche 1982; Byrne et al. 2003; Mather et al. 2010).

One skin display which is assumed to be antipredator in nature and is seen across the cephalopod groups (Moynihan 1975) is the eye spot dymantic (also called deimatic; Hanlon and Messenger 1996). A pair of eye spots is produced on the dorsal mantle of cuttlefish (Hanlon and Messenger 1988). Spots can be expressed unilaterally in cuttlefish (Langridge 2006) and dorsolaterally on squid (Moynihan and Rodaniche 1982), while a brown patch is produced around the real eyes on octopuses (Packard and Sanders 1971). This display has been seen at approach by humans and is only assumed to be antipredator, though only recently have Langridge (2006) and Langridge et al. (2007) conducted controlled laboratory testing of antipredator displays and behaviour in Sepia officinalis cuttlefish. They found a predator-specific sequence of escalating defensive responses with decreasing predator approach distance. Even though camouflage is not appropriate, would squid make these visual antipredator displays?

Evaluating the target and the function for which a response to predators is generated is often difficult. Caro (1986a, 1986b) discusses this problem for evaluation of stotting behaviour of gazelles to approach big cats, and Caro et al. (2004) do the same for comparative antipredator actions in artiodactyl mammals. In addition, the form of a display and its match to the predator situation may be more complex and variable than we assume at first sight, as Smith (1977) points out for the structure of phoebe calls and Hennessy et al. (1982) describe for situations eliciting ground squirrel tail flagging. Time may be short but the 'decisions' are not simple. The antipredator situation in open water offered an opportunity to look at this squid dymantic display in detail and properly assess its function.

Field observation of threat by predators and squid behaviour during flight decisions offers a chance to look at a simple physical situation where the flee-stay alternatives (Ydenberg and Dill 1986) are obvious. 
The jet-assisted locomotion of squid, while inefficient compared to fishes' undulation over long distances (O'Dor and Webber 1986), nevertheless produces a very fast short-range escape system. The cephalopods are also known for their intelligence (Wells 1978; Mather 1995), and therefore we can predict that they will balance the use of displays and flight depending upon the guild of fishes around them, using what Ydenberg and Dill (1986) and Owings and Morton (1998) call an assessment and management approach to both flight and communication. This article will look for such reactions in the ocean, evaluating the flight and antipredator displays of squid, mostly to approaching fish, to find such management of responses.

\section{Material and methods}

\section{Subjects}

The Caribbean reef squid (S. sepioidea) is a demersal species living in small groups near the shore but in midwater, found across the Caribbean Sea (Roper et al. 1984). The life span is approximately 1 year (LaRoe 1971). Females lay eggs in protected areas on the sea bottom but give no parental care. The young hatch at about $10 \mathrm{~mm}$ total length and move almost immediately to protected areas such as sea grass beds (Moynihan and Rodaniche 1982) or, in the case of the population observed, to protected areas near the sea surface. They grow to an adult size of $0.3 \mathrm{~m}$ length and, with a semelparous reproductive system, they show no signs of reproductive maturity until the last 6 weeks or 1 month of their lives. They are nearly always found in small groups ( $<50$, usually $<20$ in this location) in daytime, but disperse to hunt at night.

\section{Procedure}

The study was carried out at Bonaire, a small volcanic island in the southern Caribbean located at $12^{\circ} 15^{\prime} \mathrm{N}$ and $68^{\circ} 28^{\prime} \mathrm{W}$, during May and June of $1999-2003$. A narrow strip ( $<50 \mathrm{~m}$ ) of shallow water with sand, coral and coral rubble as bottom (1-8m depth) extended out from the shoreline until a steep dropoff, and squid groups were found between shore and drop-off. We observed them on the west side of the island (protected from trade wind-generated waves) along approximately $3000 \mathrm{~m}$ of shoreline and, as we worked in the area where dive resorts were spaced about every $1000 \mathrm{~m}$ along the shoreline, squid were already somewhat habituated to human activity. All of the nearshore is Bonaire Marine Park, with the result that the squid and fish are protected and the fauna is diverse and similar to that present decades ago, a contrast to the lack of large fish near other islands that is the result of subsistence fishing. Many potential predators of squid were thus present; Randall's (1967) study of the stomach contents of fish species from the Caribbean showed that many take cephalopods casually as prey. They were patrolling during the daytime, not overtly hunting but always on the alert for unwary prey.

Observation of interactions of squid with other animals were carried out by snorkellers who, in pairs, watched a group of squid for an hour at a time while floating at the water surface, keeping as still as possible so that the squid became habituated to their presence. Observers took notes on underwater slates and transferred their results to computer data files as soon after their 1-hour shift as possible. As the squid activity cycle was somewhat crepuscular (in mid-day squid were less reactive though still swimming slowly), peak activity time was between 7:00 and 10:00 and 15:00 to 18:00. Squid behaviour was disrupted by divers' lights so that no systematic observation was possible at night. Observation of displays and antipredator behaviour in 1999 was through notations by observers when the squid were not carrying on reproductive behaviour (Mather 2004), but it became more systematic as the study focused on specific questions (the data from 1999 were not included in any analyses). 
Because observation was opportunistic, individual animals were not identified. No inter-rater reliability was established but volunteer team members (mostly graduate students and colleagues) had extensive briefings, their first few days' observations were monitored by the author and their data re-checked, and evening discussions of information, variables and assignments were regular. This included, for instance, extensive ongoing discussion of identification of fish species, and although most team members were experienced divers, they also used Humann's (1994) guide to identification of Caribbean fish species.

\section{Vigilance}

For the study of vigilance, five observers spent two or three 20-min shifts each watching a specific group of subadults over 3 weeks in May 2003. Approximately 30 squid were in the area, in loosely organized small $(<12)$ mixed-sex groups at a total of eight different locations. Group membership shifted across days, and group size was relatively fluid within days, shifting with recruitment and dispersal by predators but each day squid formed into two or three groups, a few hundred meters apart. There were 96 observations of these groups for a total of $32 \mathrm{~h}$. Each observer initially recorded location, depth, group size and membership and then recorded every time the animals moved more than $1 \mathrm{~m}$. Groups all moved at once, though observers kept watch for vigilance by members at the end of the approximately linear group. See Adamo and Weichelt (1999) for evidence of this lack of individualized responses in Sepioteuthis lessoniana, despite earlier suggestions (Moynihan and Rodaniche 1982; Hanlon and Messenger 1996) that squid groups used sentinels, but video analysis was necessary to confirm this. Observers constantly scanned the surroundings and recorded the apparent source of disturbance (fish, humans, other, none observable), estimated the distance squid moved in meters (adult squid averaged $0.3 \mathrm{~m}$ total length so this assisted our calculation) and the speed (slow, medium and fast) that they moved and the change in spacing of the group (closer, same or spread further apart).

\section{Responses to fish}

For the study of responses to fish during 4 weeks of May 2000, eight observers in pairs watched a group of squid and recorded the reaction when any individual was approached by a fish, conspecific or human ( $n=927$ approaches, 27 species), with approach defined as a reduction of distance to the squid of $0.5 \mathrm{~m}$ or more. Small (<10 cm length), subadult and adult (producing reproductive behaviour sequences) squid were observed but there were few $(n=85)$ observations of the small ones. Observers noted the species approaching as well as the distance from the squid when it reacted or the closest approach if there was no reaction. In addition, we recorded the approacher's size in percentage of squid size and speed as slow, medium or fast.

Two squid reactions were taken. One was movement away approximately $180^{\circ}$ from the squid: none, slow ( $<1$ body length/sec), medium ( $1-5$ body length/sec) and fast ( $>5$ body length/sec) and a second was colour changes (none, zebra, dymantic and paling, see Byrne et al. 2003, Mather et al. 2010). These skin displays were on a continuum of reaction to disruption; the diagonal dark slashes of zebra were usually produced to conspecifics as an indication of aggression or annoyance, often by males to other males (Mather 2004). The brown paired dots of dymantic on a pale mantle were presumed to be a startle and anti-predator display (Moynihan and Rodaniche 1982) and the chromatophore contraction of paling, resulting in an over-all white colour, was a common fright response (personal observation) (Byrne et al. 2003). We observed a few responses to each of the species, but $78 \%$ of the responses were to three types of fish, herbivorous parrotfish (Scaergus sp.), which were identified to genus as the juveniles are difficult to discriminate $(n=456)$, and diurnal predators bar jack (Caranx ruber) $(n=161)$ and snapper (Ocyurus chrysurus; $n=105$ ). Thus statistical analyses were made on responses to these species. Influences of species, fish speed and fish size on squid speed of movement away were submitted to 
analysis of variance and both movement and escalation of disruption displays to stepwise multiple regression.

\section{Dymantic dot display}

For the study of dymantic dots, initial observations in 2000 were again taken while observers watched squid groups, and they noted the display in three different situations from a total of approximately 50 animals and 330 observations. Previous descriptions (Moynihan and Rodaniche 1982) had given the impression that dots were displayed as a pair on the posteriolateral mantle. Instead squid could display dots (usually a pair) on positions selected from four anterio- and posteriolateral mantle locations (Figure $1 \mathrm{a}$ and $\mathrm{b}$ ), and this finding led to further more systematic observations, starting in May 2001, of the location of dot displays when animals approached the squid. Data from these observations included noting the species approaching and direction of its approach (anterior, posterior, left, right or below). With the possibility of dots being a display to conspecifics, we also collected information about the direction of the nearest squid in 2002 and 2003 (as squid tended to swim at the same depth this could be anterior, posterior, left or right). There were 708 observations when fish approach direction was noted and 421 in which we recorded direction of both (note that as squid are an annual species, this information came from three different groups of animals). Four percent of these displays were in response to humans, $13 \%$ to conspecifics, $17 \%$ to bar jack and $56 \%$ to parrotfish.

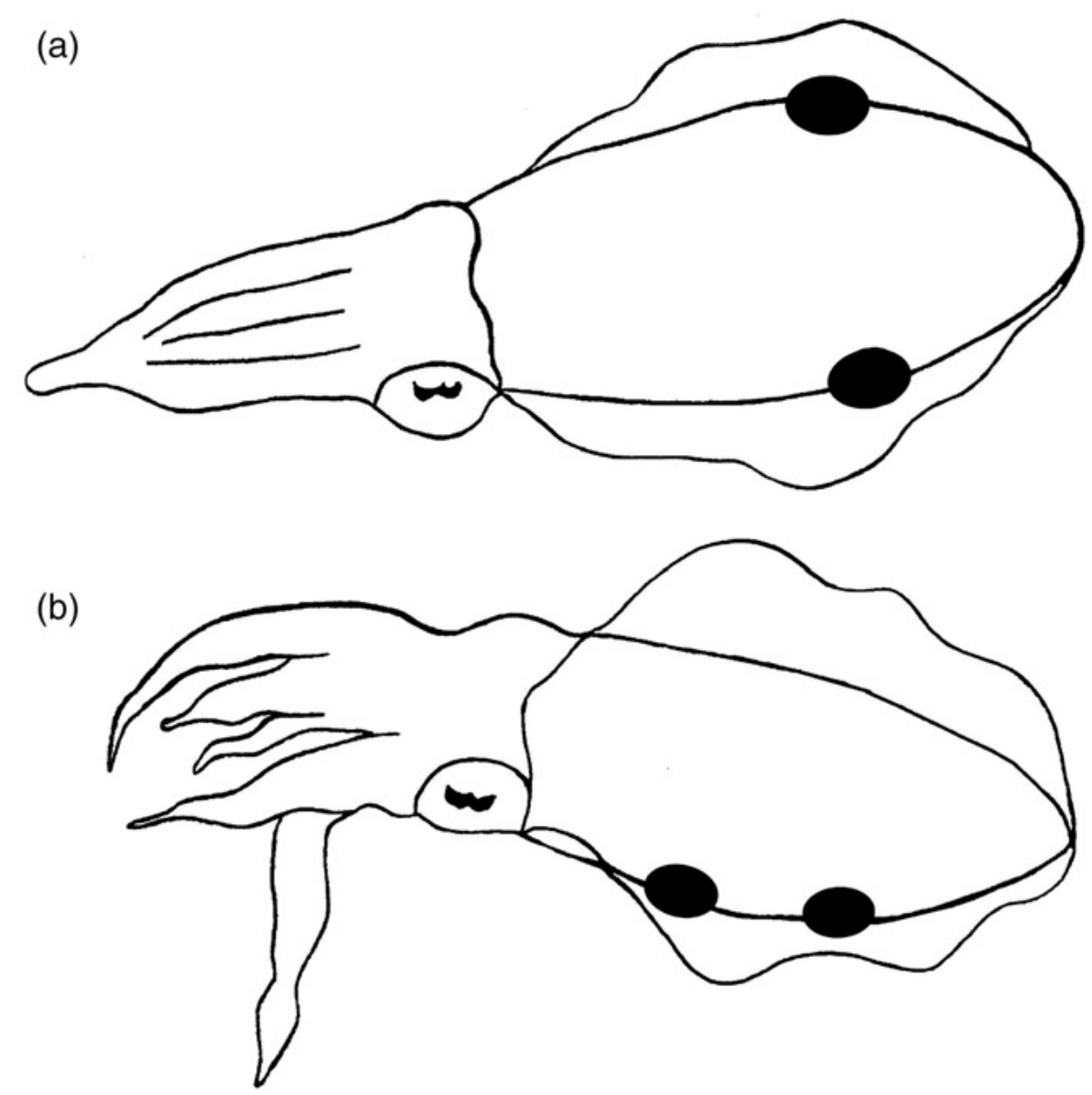

Figure 1(a). Line drawing from a photograph of a Sepioteuthis sepioidea squid with two posterior dots approaching the sea bottom. (b) Line drawing from a video of a squid displaying left anterior and posterior dots to a fish approaching from the left. 


\section{Results}

\section{Vigilance responses}

Squid groups moved a distance of more than a meter quite often, a mean of eight times per hour. When these responses were grouped across $3 \mathrm{~h}$ bins (06:00-08:59, 09:00-11:59, 12:00-14:59 and 15:0017:59), there was a difference in the response frequency across time, chi-square $(4)=6.224, p<0.05$. Moves were more frequent $(11.5 / \mathrm{h})$ in early morning, less so $(5.4 / \mathrm{h})$ in late morning and more $(7.5 / \mathrm{h})$ in the afternoon. Squid moved a mean distance of $4.5 \mathrm{~m}$, though the mode was $2 \mathrm{~m}$, and they generally returned to the location within $2 \mathrm{~min}$. When we looked at the cause of movement, in $53 \%$ of the instances, the observer could not identify it; $29 \%$ of the moves were to the approach of a potential predator including $21 \%$ to bar jacks, only $5 \%$ to the very common parrotfish and $6 \%$ to human activity such as a swimmer going by or a dive boat starting its engine. Speed of departure varied widely with $30 \%$ slow, $40 \%$ medium speed and $40 \%$ fast. A squid group seldom (6\%) spaced itself more widely at disturbance, in $47 \%$ of the instances the spacing stayed the same and in $47 \%$ the group became more compact (mean distance between individuals decreased), a significant difference, chi-square $(3)=84.5, p<0.001$. Clearly, squid groups often moved from their position, though the source of disturbance was not always easy to pinpoint.

Table 1. Characteristics of approach of three fishes.

\begin{tabular}{|llccc|}
\hline Characteristic & & Parrotfish (\%) & Bar Jack (\%) & Snapper (\%) \\
\hline Size (in squid length) & Mean & 1.3 & 1.7 & 1.5 \\
& Mode & 1.0 & 1.0 & 1.2 \\
Speed of approach & Fast & 60 & 25 & 76 \\
& Medium & 26 & 60 & 20 \\
& Slow & 14 & 14 & 4 \\
Closest approach & $<1 \mathrm{~m}$ & 74 & 17 & 9 \\
& $1-2 \mathrm{~m}$ & 18 & 21 & 29 \\
& $2-3$ & 4 & 16 & 33 \\
& $>3 \mathrm{~m}$ & 4 & 47 & 29 \\
\hline
\end{tabular}

Notes: Parrotfish $(n=456)$; bar jack $(n=161)$ and snapper $(n=105)$ that elicited responses from squid.

\section{Response to fish approaches}

When responses to the three common fish that approached were compared, several differences emerged. An analysis of variance looking at influences on speed of movement was significant, $F(2719)=155.82, p=0.001$, squid moved away to fast approaching snapper and parrotfish but not bar jack (Table 1) and to closer parrotfish than barjack. Because of the lack of normal distributions in responses, the differences in colour changes to the three fish species were compared by a chi-square test but were also significant, chi-square $(12)=171.59, p<0.001$. Squid often made no colour change to parrotfish, seldom made a zebra response to bar jack and either ignored a snapper or paled at its approach (Table 2). A separate regression analysis was carried out for the different fish species to see which characteristic of the approach (speed, distance or fish size) predicted an escalation of movement and colour change. For pattern change to parrotfish, the first variable to enter was Distance, $F=15.481, p<0.001$, and the second was Size, $F=10.591, p<0.001$. The same variables affected movement, distance was first at 
$F=284.299, p<0.001$ and size was second at $F=136.907, p<0.001$. This pattern was in contrast with that for snapper in which pattern change, $F=5.592, p<0.002$, and movement, $F=4.449, p=0.006$ were both predicted by speed, although a second step was added for the effect on movement, distance at $F=4.402$, $p<0.001$. The prediction of reaction to bar jack was more like that for parrotfish, pattern change by distance, $F=13.032, p<0.001$ and first distance, $F=16.808, p<0.001$ and also dize $F=10.160, p<0.001$, for movement. Squid were clearly responding to different cues about the different approaching fish species, and these reactions were not significantly different across squid size groups.

\section{Dymantic dot displays}

Dot displays were produced in three different situations: while the squid were casually hunting schools of small fish or when they were approaching the bottom, and reactively when a fish, human or conspecific approached. The dots produced in the first two situations were almost always the posteriolateral pair, directionality was only a feature of the reactive fish-approach situation (Figure 1). The probability of a different selection of dot locations being produced was significantly different across conditions, chi-square $(12)=26.8, p<0.001$ (Table 3).

Table 2. Responses of squid to approach of three fishes.

\begin{tabular}{|llccc|}
\hline Response & & Parrotfish (\%) & Bar Jack (\%) & Snapper (\%) \\
\hline Move & None & 74 & 28 & 7 \\
& Avoid & 17 & 21 & 46 \\
& Medium & 8 & 46 & 44 \\
& Fast/Far & 1 & 6 & 4 \\
Colour change & None & 48 & 27 & 33 \\
& Zebra & 15 & 2 & 0 \\
& Dots & 20 & 13 & 0 \\
& Pale & 17 & 58 & $67 \%$ \\
\hline
\end{tabular}

Notes: Parrotfish ( $n=456)$, bar jack $(n=161)$ and snapper $(n=105)$ in movement (none, avoid, medium and fast/far) and skin displays (none, Zebra, Dots and Pale).

Table 3. Location of eye-spot dymantic dots (usually two) on the dorsal surface of the mantle of squid in three different situations.

\begin{tabular}{|lcccc|}
\hline & & \multicolumn{3}{c|}{ Dots } \\
\cline { 3 - 5 } Direction of approach & Left anterior & Left posterior & Right anterior & Right posterior \\
\hline Approach & 32 & 38 & 186 & 196 \\
Hunting & $1^{\mathrm{a}}$ & $1^{\mathrm{a}}$ & 55 & 54 \\
Near substrate & $3^{\mathrm{a}}$ & $3^{\mathrm{a}}$ & 46 & 47 \\
\hline
\end{tabular}

Notes: At the approach of an animal ( $n=228)$; when hunting small fish apart from the group $(n=55)$ and when approaching the sea bottom ( $<0.3 \mathrm{~m} ; n=47) .{ }^{\text {aAll }}$ four dots.

Fish or other animals approached from all directions; the likelihood of different dots being produced at approaches from above and below was used as the comparison probability for approaches in the horizontal plane. A chi-square test showed that dots produced at approaches on the same plane as the squid were significantly different from above-below, chi-square $(16)=579.8, p<0.001$ (Table 4). Dot 
displays were directional in nature. Squid were much more likely to display the left anterior dot when approaches were from the left or the anterior and less likely to show the right posterior one when an approach was from the left. They were more likely to display the right anterior and right posterior dot with an approach from the right and less likely to show the left posterior one. As squid seldom swam above or below one another this default comparison could not be used for dot production towards conspecifics, the probability of dot directions with reference to the position of the nearest squid was compared again with the mean for all conditions. They were not significantly different, chi-square $(16)=8.1$, NS (Table 5). The dot selection by directionality thus supports a hypothesis that the dymantic dots were addressed to the potential predator and not to conspecifics as warning.

Table 4. Location of eye-spot dymantic dots displayed on the dorsal surface of the mantle of squid when other animals approached them from different directions.

\begin{tabular}{|lcccc|}
\hline & \multicolumn{4}{c|}{ Dots } \\
\cline { 2 - 5 } Direction of approach & Left anterior & Left posterior & Right anterior & Right posterior \\
\hline Above/below $(n=168)$ & 3 & 148 & 51 & 131 \\
Anterior $(n=84)$ & $16^{\mathrm{a}}$ & 72 & 11 & 71 \\
Right $(n=156)$ & 3 & $50^{\mathrm{a}}$ & $30^{\mathrm{a}}$ & $150^{\mathrm{a}}$ \\
Posterior $(n=134)$ & 5 & 121 & 6 & 127 \\
Left $(n=166)$ & $28^{\mathrm{a}}$ & 135 & 11 & 51 \\
\hline
\end{tabular}

Notes: Above/below as comparison with left, right, anterior and posterior. ${ }^{2}$ Different from above/below.

Table 5. Location of eye-spot dymantic dots displayed on the dorsal surface of the mantle of squid after the approach of another animal with reference to the direction to the nearest conspecific.

\begin{tabular}{|lcccc|}
\hline & \multicolumn{4}{c|}{ Dots } \\
\cline { 2 - 5 } Direction of approach & Left anterior & Left posterior & Right anterior & Right posterior \\
\hline Anterior $(n=28)$ & 2 & 4 & 22 & 21 \\
Right $(n=160)$ & 6 & 10 & 135 & 125 \\
Posterior $(n=75)$ & 8 & 6 & 55 & 56 \\
Left $(n=75)$ & 10 & 11 & 123 & 140 \\
\hline
\end{tabular}

\section{Discussion}

Squid certainly have a situation in which, with eight flights per hour, they make 'decisions' (Ydenberg and Dill 1986) to flee. But even though squid only fed casually in the daytime, there was no mixed motivation to feed or to flee (e.g. Lendrem 1983), and no variable habitat to choose from (e.g. Stein and Magnuson 1976), squid did not appear to make automatic decisions in this situation. Different fish species were not only allowed to approach to different distances, but also the main cue that triggered an escalated response by squid was different, even in the two species of predatory fish for which there were enough data points. Redshank birds show a similar array of responses to different predators (Cresswell 1993). Perhaps squid had learned which species was a serious threat and which could be tolerated, although recruitment of different components of the reaction was also true for young cuttlefish (Hanlon and Messenger 1988), and lab-reared ones (Langridge 2006). Such learning may be generally true, as Diana monkeys apparently learn that chimpanzees alarm call to leopards are indicative of predator threat (Zuberbühler 2000) and fathead minnows update predator information over time (Ferrari and Chivers 2006). 
Antipredator behaviour is difficult to study in the natural environment. As Boland (2003) observed, predation events are rare in nature; in 5 years of observation we saw three; one by a lizardfish, one by a grouper as a female was laying eggs and one by a larger conspecific. The casual nature of much of the observation and the lack of inter-rater reliability checks, as well as the variation in such environmental conditions as current, water clarity and depth, means that the present field observations must be evaluated with these constraints in mind. Still, many measurements such as fish size in relation to squid size and location of dot displays were easy for observers to calculate. Careful laboratory studies such as those of Langridge (2006) for cuttlefish would be an important addition to these observations. However, open-ocean squid are difficult to keep in the laboratory and laboratory situations are inevitably much simpler than the natural environment. Observing what animals actually do where they live has constraints, but it also has strengths in terms of applicability to real-world situations.

On what basis might the squid discriminate amongst the different species? As cephalopods generally have excellent vision (Budelmann 1994; Gleadall and Shashar 2004), visual cues, perhaps about fish size and shape, should be used in assessment. The predictors of increased size and closer distance that squid use to escalate escape behaviour from parrotfish and bar jacks suggest an increase in the size of the retinal image of the approaching fish as a triggering stimulus. But squid also have a lateral line analogue that is sensitive to even very small mechanical deformations of the water around them (Budelmann 1994). Observers often commented about approaches by fast-swimming predators such as the snapper that the squid left first and, several seconds later, the observer saw the fish from afar. As the speed of approach was the predictor of whether to escalate response to snapper, vibro-tactile cues may also play a part. Different fish species have different body-tail undulation patterns (see Alexander 2003, for fish swimming), producing different water movement patterns which could be used as cues. Slowdispersing chemical cues such as those used by fish (Hawkins et al. 2004; Ferrari and Chivers 2006) did not appear to be a factor in this environment as reaction times were too fast.

Why might squid react to herbivorous parrotfish, who were clearly no predatory threat, and indeed often ignored? Perhaps, they had tuned vigilance to such a height that an approaching fish, regardless of species, sometimes caused them to spook. But also parrotfish are large and heavy, perhaps the marine equivalent of cows for people. If one of them is coming at you quickly, it is logical to move out of the way, even if it is not likely to eat you.

That squid significantly often closed up in the group as a result of fish approach is a reminder that each squid is not a completely separate entity in its response. Almost nothing is known about squid grouping except that it is obligate in the daytime, but these squid appear to be forming schools by Ritz's (1994) definition of attraction to one another (Boom et al. 2001). Closing up of a group is also common for fish schools under threat of predation (Pitcher 1986) and probably makes predation more difficult by generating a confusion effect (Neal and Cullen 1974).

The production of a dymantic dot display (note that eye spots seem to be conserved as an anti-predator display across many animal groups, see Coss and Goldthwaite 1995) is also differential in interesting ways. Eye spot production seemed to be a proactive response particularly when squid were approaching the bottom, a place that may have an abundance of predators in hiding. Yet, it was also directional and a reactive response to some but not all approaching fish, a variation of display structure reminiscent of ground squirrel tail flagging (Hennessey et al. 1982) and phoebe calls (Smith 1977). Simply saying that the dymantic dots are an anti-predator display does not define the situational and species variables that control their production. Squid never displayed them to snapper, for instance, and Thomson's gazelles similarly used predator inspection only for some potential predators (FitzGibbon 1994). As Ydenberg and Dill (1986) point out, predator recognition does not always correlate with flight. 
As squid and cuttlefish can clearly use the skin display system unilaterally (Hanlon and Messenger 1996; Langridge 2006), why would squid have been only statistically likely to make dots in the direction of approaching fish? One possibility is that the bilateral brain organization of cephalopods (Young 1971) might lead to lateralized output and make left-right differentiation easy but anteroposterior differentiation more difficult. Another is that the lateralization of output develops with maturity to facilitate the use of sexual displays at the end of the lifespan. The dots were not a response of very young squid, who instead use a variety of camouflage displays (Moynihan and Rodaniche 1982; Mather et al. 2010), and subadults might still be developing display directionality. A third possibility comes from observation of dots displays to bar jack. Sometimes, instead of selecting dots that were directional towards the fish, a squid would pivot and tilt so that its dorsal surface was facing towards the fish and produce the two posterior dots, a different way of generating display directionality.

Squid reaction to bar jack (also observed by Hanlon, in Hanlon and Messenger 1996) is surprisingly differential. While this species is potentially a predator and on average squid did not allow it to approach closely; nevertheless, bar jack often approached squid to well within a metre and the animal often simply gave them a dymantic dot response. Other animals may allow close approach of some potential predators or even move to examine them, and FitzGibbon (1994) showed that such predator approach to cheetahs by gazelles seemed to have a benefit in alerting, allowing learning about predators and perhaps letting predators know that they were detected. Not all potential predators are suitable for inspection; gazelles did not approach spotted hyaenas (FitzGibbon 1994) and squid did not allow near approaches by snapper or other large fast-moving predatory fish such as horse-0eyed jack, barracuda and tarpon. What looks like a simple situation of predator approach may elicit complex reactions, depending on the predator.

While squid vigilance and antipredator behaviour during approaches in an open habitat, as for those of deer (Lingle and Wilson 2001) and emus (Boland 2003) in prairie, seems a simple situation, the response is still complex. This complexity is normal; Caro (2005, p. 447) talks of prey species using a 'menu of defenses' even against one predator and Langridge et al. (2007) describe the modulation of cuttlefish responses by both threat level and contextual variables. We have not begun to assess the effect of group size of squid on vigilance, which is found in other species (Lima 1995). Such vigilance should also be much diminished when squid are engaged in courtship behaviour, a universal tradeoff for animals. An assessment of the development of the dymantic dot patterns should help us understand its function better. Squid do indeed have complex decision making (sensu; Ydenberg and Dill 1986) about flight and its alternatives such as skin displays in the face of predator threat, and further investigation of this system should be rewarded by deeper understanding of its variety and functions, and what that may mean to their flexible decision-making processes.

\section{Acknowledgements}

Thanks to the Squid Chasers, my volunteer team members over the years of the study, and to the staff members of the dive centres of Bonaire who made our study easier. A special thanks goes to my husband Lynn Mather for team management and Ruth Bergen Braun for manuscript preparation.

\section{References}

Adamo SJ, Weichelt KJ. 1999. Field observations of schooling in the oval squid Sepioteuthis lessoniana. J Mollusc Stud. 65:377-380. Alexander RM. 2003. Principles of animal locomotion. Princeton: Princeton University Press. 
Boland CRJ. 2003. An experimental test of predator detection rates using groups of free-living emus. Ethology. 109:209-222.

Boom S, Byrne RA, Mather JA. 2001. Schooling behaviour of the Caribbean reef squid Sepioteuthis sepioidea in Bonaire. Paper presented at the XXVII Ethological Conference, August.

Budelmann BU. 1994. Cephalopod sense organs nerves and the brain: adaptations for high performance life style. Mar Freshwater Behav Physiol. 25:13-33.

Byrne RA, Griebel JB, Wood JB, Mather JA. 2003. Squid say it with skin: a graphic model for skin displays in Caribbean reef squid (Sepioteuthis sepioidea). Berl Palaobiol Abh. 3:29-35.

Caro TM. 1986a. The functions of stotting: a review of the hypothesis. Anim Behav. 34:649-662.

Caro TM. 1986b. The functions of stotting in Thomson's gazelles: some tests of the predictions. Anim Behav. 34:663-684.

Caro TM. 2005. Antipredator defenses in birds and mammals. Chicago: University of Chicago Press.

Caro TM, Graham CM, Stoner CJ, Vargas JK. 2004. Adaptive significance of antipredator behavior in artiodactyls. Anim Behav. 67:205-228.

Coss RG, Goldthwaite RO. 1995. The persistence of old designs for perception. In: Thompson NS, editor. Perspectives in ethology. Vol 11: behavioral design. New York: Plenum. p. 83-148.

Cresswell W. 1993. Escape response by redshanks Tringia totanus on attack by avian predators. Anim Behav. 46:609-611.

Dugatkin LA. 2004. Principles of animal behavior. New York: Norton.

Ebensperger LA, Hurtado MJD. 2005. On the relationship between herbaceous cover and vigilance activity of degus (Octodon degus). Ethology. 111:593-608.

Ferrari MCO, Chivers DP. 2006. Learning threat-sensitive predator avoidance: how do fathead minnows incorporate conflicting information? Anim Behav. 71:19-26.

FitzGibbon CD. 1994. The costs and benefits of predator inspection behaviour in Thomson's gazelles. Behav Ecol Sociobiol. 34:139-148.

Gleadall IG, Shashar N. 2004. The octopus's garden: the visual world of cephalopods. In: Prete FR, editor. Complex worlds from simpler nervous systems. Cambridge: MIT Press. p. 269-307.

Hanlon RT, Forsythe JW, Joneschild DE. 1999. Crypsis conspicuousness mimicry and polyphenism as antipredator defenses of foraging octopuses on Indo-Pacific coral reefs with a method of quantifying crypsis from video tapes. Biol J Linnaean Soc. 66:1-22.

Hanlon RT, Messenger JB. 1988. Adaptive colouration in young cuttlefish (Sepia officinalis): the morphology and development of body patterns and their relation to behaviour. Philos Trans $\mathrm{R}$ Soc London [Biol]. 320:437-487.

Hanlon RT, Messenger JB. 1996. Cephalopod behaviour. Cambridge: Cambridge University Press. 
Hawkins LA, Magurran AE, Armstrong JD. 2004. Innate predator recognition in newly-hatched Atlantic salmon. Behaviour. 141:1249-1262.

Heatwole H. 1968. Relationship of escape behavior and camouflage in Anoline lizards. Copeia. 1:109113.

Hennessey DF, Owings DH, Rowe MP, Coss RG, Leger DW. 1982. The information afforded by a variable signal: constraints on snake-elicited tail flagging by California ground squirrels. Behaviour. 78:188-226.

Humann P. 1994. Reef fish identification: Florida Caribbean Bahamas. Jacksonville: New World Publications.

Langridge KV. 2006. Symmetrical crypsis and symmetrical signaling in the cuttlefish Sepia officinalis. Proc. R. Soc. B. 273:959-967.

Langridge KV, Broom M, Osorio D. 2007. Selective signalling by cuttlefish to predators. Curr Biol. 17:R1044-R1045.

LaRoe ET. 1971. The culture and maintenance of the Loliginid squids Sepioteuthis sepioidea and Doryteuthis plei. Mar Biol. 9:9-2.

Lendrem DW. 1983. Predation risk and vigilance in the blue tit (Parus caerulus). Behav Ecol Sociobiol. 14:9-13.

Lima SL. 1995. Back to the basics of anti-predatory vigilance: the group-size effect. Anim Behav. 49:1120.

Lima SL, Valone TJ. 1986. Influence of predation risk on diet selection: a simple example in the grey squirrel. Anim Behav. 34:536-544.

Lingle S, Wilson WF. 2001. Detection and avoidance of predators in white-tailed deer (Odocoileus virginianus) and mule deer (O. hemionus). Ethology. 107:125-147.

Mather JA. 1995. Cognition in cephalopods. Adv Stud Behav. 24:316-353.

Mather JA. 2004. Cephalopod skin displays from concealment to communication. In: Oller K, Greibel U, editors. Evolution of communication systems. Cambridge: MIT Press. p. 193-213.

Mather JA, Byrne RA, Greibel U. 2010. Squid dances, movements and postures of Sepioteuthis sepioidea with a muscular hydrostat system. Mar Freshw Behav Physiol. 43: 45-61.

Messenger JB. 2001. Cephalopod chromatophores: neurobiology and natural history. Biol Rev. 76:473528.

Moynihan $\mathrm{MH}$. 1975. Conservatism of displays and comparable stereotyped patterns among cephalopods. In: Baerends FRS, Beer G, Manning A, editors. Function and evolution in behaviour, essays in honour of Professor Niko Tinbergen. Oxford: Oxford University Press. p. 276-291.

Moynihan MH, Rodaniche AF. 1982. The behaviour and natural history of the Caribbean reef squid Sepioteuthis sepioidea with a consideration of social signal and defensive patterns for difficult and dangerous environments. Adv Ethol. 125:1-150. 
Neal SR, Cullen JM. 1974. Experiments on whether schooling by their prey affects the hunting of cephalopods and fish predators. J Zool London. 172:549-569.

O'Dor RK, Webber DM. 1986. The constraints on cephalopods: why squid aren't fish. Can J Zool. 64:1591-1605.

Owings DH, Morton ES. 1998. Animal vocal communication: a new approach. Cambridge: Cambridge University Press.

Packard A. 1995. Organization of cephalopod chromatophore systems: a neuromuscular imagegenerator. In: Abbott NJ, Williamson R, Maddock L, editors. Cephalopod neurobiology. Oxford: Oxford University Press. p. 331-367.

Packard A, Sanders GD. 1971. Body patterns of Octopus vulgaris and maturation of the response to disturbance. Anim Behav. 19:780-790.

Pitcher TJ. 1986. Function of schooling behavior. In: Pitcher TJ, editor. The behavior of teleost fishes. Baltimore: Johns Hopkins University Press. p. 294-338.

Randall JE. 1967. Food habits of reef fishes of the West Indies. Stud Trop Oceanogr. 5:665-847.

Ritz DA. 1994. Social aggregation in pelagic invertebrates. Adv Mar Biol. 30:156-216.

Roper CFE, Sweeney MJ, Naun CE. 1984. FAO species catalogue 3. Cephalopods of the World FAO Synopsis. 125 Vol. 3, p. 277.

Smith WJ. 1977. The behavior of communicating: an ethological approach. Cambridge: Harvard University Press.

Stein RA, Magnusson JJ. 1976. Behavioral response of a crayfish to a fish predator. Ecology. 57:751761.

Wells MJ. 1978. Octopus: behaviour and physiology of an advanced invertebrate. London: Chapman \& Hall.

Ydenberg RC, Dill LM. 1986. The economics of fleeing from predators. Adv Stud Behav. 16:229-249.

Young JZ. 1971. The anatomy of the nervous system of Octopus vulgaris. Oxford: Clarendon. Zuberbu" hler K. 2000. Causal knowledge of predators' behaviour in wild Diana monkeys. Anim Behav. 59:209220. 